\title{
In-situ Micromechanical Testing of Neutron Irradiated FeCrAl Alloys
}

Keyou Mao ${ }^{1}$, Gussev Maxim ${ }^{1}$, Caleb Massey ${ }^{1}$, Kinga Unocic ${ }^{1}$, Philip Edmondson ${ }^{1}$ and Kevin Field ${ }^{2}$

${ }^{1}$ Oak Ridge National Laboratory, Oak Ridge, Tennessee, United States, ${ }^{2}$ University of Michigan, Ann Arbor, Michigan, United States

Iron-Chromium-Aluminum (FeCrAl) alloys have been selected as one of the candidate accident tolerant fuels (ATFs) claddings to replace Zirconium-based alloys of the current fleet of light water reactors (LWRs). FeCrAl alloys have several advantages such as reasonable irradiation resistance [1,2], superior oxidation and corrosion tolerance [3], as well as good processing and weldability [4]. As a body-centeredcubic (bcc) type alloy, the activation of dislocation slip to which plane is not well defined [5] and the localized strain burst is driven by screw dislocations [6]. After irradiation, a combination of dislocation channeling [7] and strain bursts [8] are affected by irradiation-induced defects. Prior tensile studies of $\mathrm{FeCrAl}$ alloys have demonstrated irradiation-induced hardening behavior, but the detailed analysis on the dislocation structure after deformation and the chemical composition have not been fully performed. Due to all these complexities, this study aims to understand the role of irradiation-induced defects on the mechanical response and deformation mechanisms in the $\mathrm{FeCrAl}$ alloy system.

This work focuses on a FeCrAl alloy of Fe-13.0Cr-5.3Al-2.0-Mo-0.1Si-0.05Y (wt.\%). The specimen was irradiated in the Oak Ridge National Lab (ORNL) High Flux Isotope Reactor (HFIR) at $\sim 282{ }^{\circ} \mathrm{C}$ to a dose of $~ 7$ dpa. Tescan MIRA3 GMH equipped with an electron backscatter diffraction (EBSD) system (Oxford Instruments) was used for scanning electron microscopy (SEM) imaging and transmission Kikuchi diffraction (tKD). A MZ.Sb (Kammrath and Weiss Tech., Germany) miniature $5 \mathrm{kN}$ tensile frame was used for SEM in-situ microtensile test at room temperature with a strain rate of $\sim 10^{-3} \mathrm{~s}^{-1}$. A FEI (now Thermo Fisher Scientific) Versa 3D dual-beam SEM/focused ion beam (FIB) was used to extract FIB liftouts from both specimens before and after micromechanical testing for transmission electron microscopy (TEM) characterization. Dislocations and loops were imaged by the JEOL JEM-2100F field emission gun scanning transmission electron microscope (FEG-STEM). Chemical composition was mapped by the dispersive X-ray spectroscopy (EDS) in a FEI Talos F200X FEG-STEM.

Figure 1(a) shows the deformed region of the unirradiated specimen near the necking region. A tkD map in Figure 1(b) indicates the grain structure and grain boundaries after tensile test with dislocation patterning (Figure 1(a)) inside one of the grains near a low-angle grain boundary. After neutron irradiation, loops and Cr-enriched $\alpha$ ' precipitates (Figure 2(a-b)) form as obstacles for dislocation motion, enabling the switch of slip activation and the sweeping of defects for dislocation-free channel formation. The role of grain rotation, interaction between irradiation-induced defects and dislocations on the channeling formation and localized strain bursts heaviliy alter the mechanical behavior [9]. 

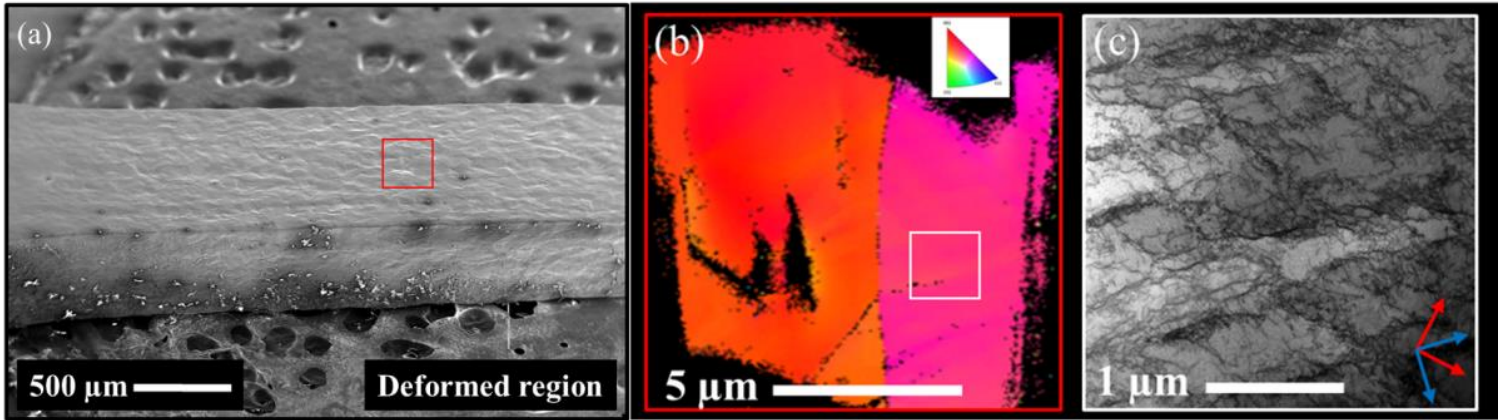

Figure 1. (a) Deformed unirradiated FeCrAl specimen. (b) Grain structure from red box in (a). (c) Dislocations from white box in (b). Red and blue arrows represent the and, respectively.

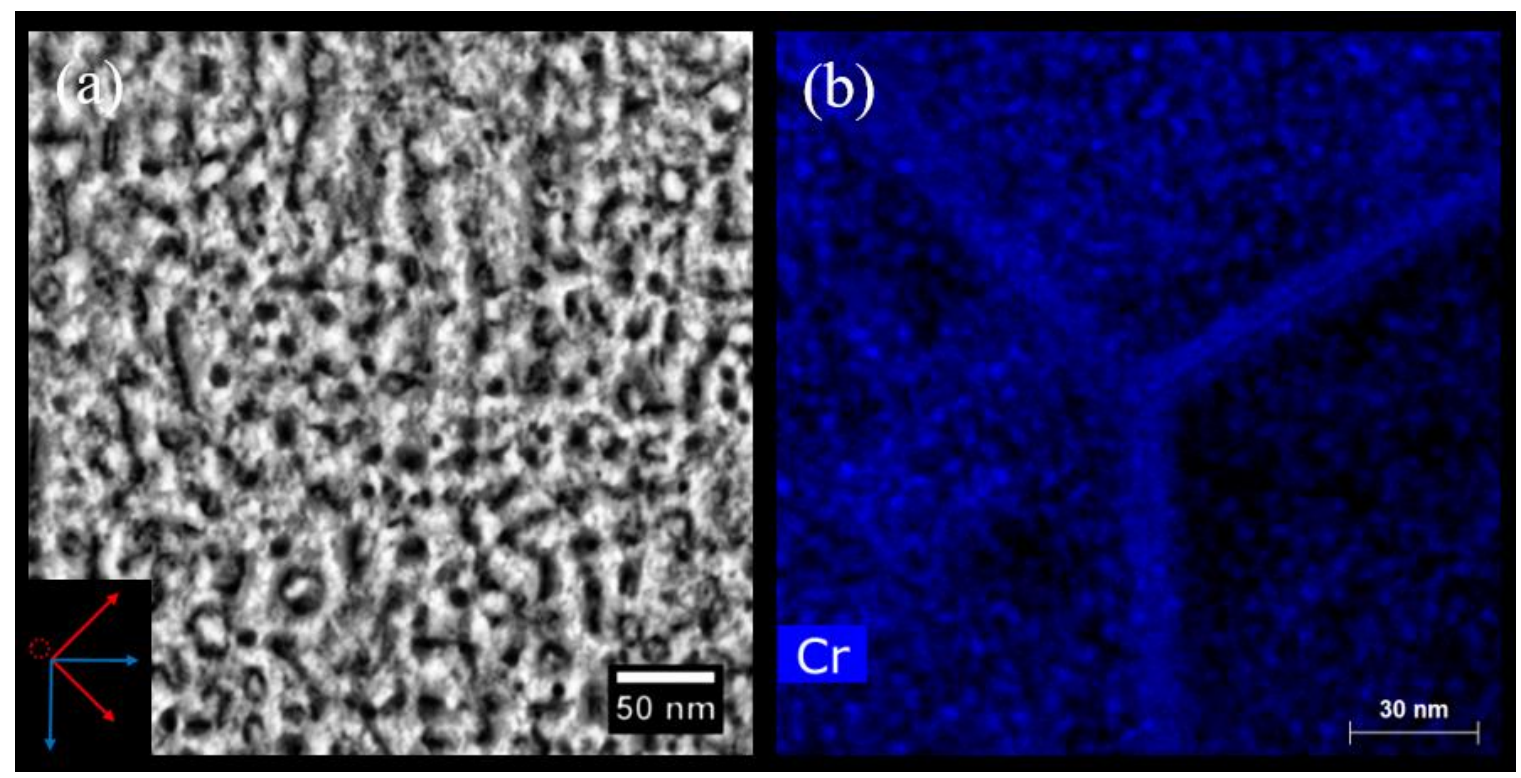

Figure 2. (a) Dislocation loops after irradiation. (b) Cr-enriched grain boundary a' precipitates. Red, blue arrows and dashed circle indicate the , and in-plane loops, respectively.

\section{References}

[1] SA Briggs et al, Acta Materialia 129 (2017), p. 217-228.

[2] KG Field et al, Journal of Nuclear Materials 465 (2015), p. 746-755.

[3] BA Pint et al, Journal of Nuclear Materials 440 (2013), p. 420-427.

[4] D Zhang et al, Journal of Nuclear Materials 527 (2019), p. 151784.

[5] CR Weinberger, BL Boyce and CC Battaile, International Materials Reviews 58 (2013), p. 296-314.

[6] Y Cui et al, International Journal of Plasticity 124 (2020), p. 117-132.

[7] A Patra and DL McDowell, Acta Materialia 110 (2016), p. 364-376.

[8] Y Cui, G Po and N Ghoniem, Acta Materialia 132 (2017), p. 285-297.

[9] The authors acknowledge funding Department of Energy (DOE) Office of Nuclear Energy, Advanced Fuel Campaign of the Nuclear Technology R\&D program. Neutron irradiation of FeCrAl alloys at ORNL's HFIR user facility was sponsored by the Scientific User Facilities Division, Office of Basic Energy Sciences, DOE. Dr. A.T. Nelson, Dr. Y. Yamamoto and Dr. D. Zhang are thanked for their many useful discussions and contributions to this work. 